Prólogo

\section{EL DIÁLOGO CONTINÚA: DESCOLONIZACIÓN DE LA CIENCIA DE LOS DESASTRES EN LATINOAMÉRICA Y EL CARIBE}

Aracely Martínez Rodas ${ }^{1 *}$, Claudia González-Muzzio ${ }^{2}$ y Victor Marchezini ${ }^{3}$

\section{RESUMEN}

El volumen dos del número especial "Descolonización de la ciencia de los desastres: enfoques desde Latinoamérica y el Caribe” (Vol. 6. Núm. 1) busca promover el diálogo para descolonizar los estudios de desastres y trascender a los enfoques, fuentes, conceptos, metodologías, valores y lenguajes occidentales 'bien establecidos' que son predominantemente ajenos a los puntos críticos de desastres y riesgos que afectan a la región. Algunos de los temas abordados en el número especial incluyen: pensamiento social latinoamericano ante los desastres y epidemias; cosmovisiones, conocimiento de los pueblos oprimidos y saberes alternativos en la investigación de desastres; ecología de los saberes; antropología de los desastres; sociología de los desastres; modelos de desarrollo y producción social de los riesgos de desastres; procesos de vulneración, formas de opresión, desigualdad y pobreza en los desastres; desastres olvidados y pueblos oprimidos; dimensiones del poder en la producción social de los riegos y en los desastres; entre otros.

\section{PALABRAS CLAVES}

Descolonización, Ciencia de los desastres, Riesgo, Amenazas, Latinoamérica, El Caribe

\section{THE DIALOGUE CONTINUES: DECOLONIZATION OF DISASTER SCIENCE IN LATIN AMERICA AND THE CARIBBEAN}

\section{ABSTRACT}

The second special issue "Decolonization of disaster science: Approaches from Latin America and the Caribbean" (Vol. 6, No. 1) seeks to promote the dialogue to decolonize disaster studies and transcend 'well-established' Western approaches, sources, concepts, methodologies, values and even languages, which are predominantly foreign views to the disaster and risk hotspots affecting the region. Some of the topics addressed in the special issue include: Latin American social thought in the face of disasters and epidemics; worldviews, knowledge of oppressed peoples and alternative knowledge in disaster research; ecology of knowledge; anthropology of disasters; sociology of disasters; development models and social production of disaster risks; processes of violation, forms of oppression, inequality and poverty in disasters; 'invisible' disasters and oppressed peoples; dimensions of power in the social production of risks and disasters; among others.

\section{KEYWORDS}

Decolonization, Disaster science, Risk, Hazards, Latin America, The Caribbean

\author{
1. Maestría en Desarrollo, \\ Universidad del Valle de \\ Guatemala, Ciudad de \\ Guatemala, Guatemala. \\ 2. Ámbito Consultores, \\ Santiago, Chile. \\ 3. Centro Nacional de \\ Monitoramento e Alertas de \\ Desastres Naturais CEMADEN, \\ São Paulo, Brasil.
}

*Autor de correspondencia: ajmartinez@uvg.edu.gt

DOI:

https://doi.org/10.55467/ reder.v6i1.89

\section{RECIBIDO}

15 de noviembre de 2021

\section{PUBLICADO}

1 de enero de 2022

\section{Formato cita}

Recomendada (APA):

Martínez Rodas, A., González-Muzzio, C. \& Marchezini, V. (2022). El Diálogo Continúa: Descolonización de la Ciencia de los Desastres en Latinoamérica y el Caribe. Revista de Estudios Latinoamericanos sobre Reducción del Riesgo de Desastres REDER, 6(1), 1-8. https://doi.org/10.55467/ reder.v6i1.89

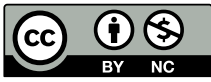

Todos los artículos publicados en REDER siguen una política de Acceso Abierto y se respaldan en una Licencia CreativeCommons Atribución-NoComercial 4.0 Internacional.

Revista de Estudios Latinoamericanos sobre Reducción del Riesgo de Desastres (REDER)

Diseño: Lupe Bezzina 


\section{¿QUÉ ES LA DESCOLONIZACIÓN?}

Como científicos y académicos, generalmente nos aproximamos a las comunidades y poblaciones con las que trabajamos desde el desconocimiento sobre sus dinámicas culturales y con prejuicios establecidos por la sociedad hegemónica en la que nos formamos y convivimos, que privilegia lo "occidental" u originado en el Norte global. Esas ideas preconcebidas nos llevan a generar conceptos sobre lo que se considera como "bienestar" desde arriba, o lo que "debe ser", y desde afuera, o sintiéndonos especialistas sobre lo que "ellos necesitan". Lo anterior nos impide conocer a las poblaciones, dialogar con ellas e incluirlas como co-partícipes de los procesos de investigación e intervención para el cambio.

Son ideas que derivan de un "hacer ciencia" desde el Norte global, donde la producción de conocimiento científico es anterior a la realizada desde Latinoamérica. En dicha región, los esfuerzos científicos iniciaron después de la Segunda Guerra Mundial, vinculados a los niveles de desarrollo de los países y con el apoyo financiero e institucional de organismos de cooperación (Santa y Herrero Solana, 2010).

En ese sentido, la producción científica latinoamericana presenta las siguientes características: escasa inversión en investigación y desarrollo, políticas públicas que no toman en cuenta investigaciones y evidencia para el desarrollo nacional, financiación escasa e inestable debido a las condiciones económicas de los países, ejecutada principalmente por el sector público y escasa población de investigadores en diferentes ciencias para realizar estudios de calidad (Santa y Herrero Solana, 2010, p. 381). Lo anterior denota una dependencia generalizada del Norte global para producir conocimiento desde el Sur global, por lo que podemos hablar de una ciencia "colonizada" que se posiciona hegemónicamente sobre otras formas de producir conocimiento. Dicha hegemonía colonial es transversal al Estado, y se evidencia en otros ámbitos institucionales, como la gestión pública, que trataremos más adelante.

La "colonización" de la ciencia y de otros ámbitos se replica al interior de nuestras sociedades. Así como el Norte global influye y determina la producción científica en el Sur global, aquellos que tenemos el privilegio de hacer ciencia reproducimos las prácticas coloniales en las comunidades y poblaciones con las cuales trabajamos. De tal manera, esa "superioridad hegemónica" desde el centro de nuestros países (generalmente las ciudades capitales o aquellas con mayor desarrollo) se traslada a la periferia con ideas preconcebidas sobre el otro, entendido como aquellas poblaciones diferenciadas por etnia, clase, ubicación geográfica, religión u otras variables, que son poco conocidas por el área central, marginadas y sobre las cuales se realizan las iniciativas de desarrollo.

Desde esta perspectiva, las iniciativas no son pertinentes culturalmente, ni toman en cuenta las dinámicas, los conflictos, los diferentes intereses y el conocimiento propio de las poblaciones a quienes van dirigidas. Por lo general, se obvia la participación de expertos locales y el diálogo con las comunidades para el co-diseño de las intervenciones, de manera que en ellas no se incluyen sus necesidades, prioridades, experiencias y formas tradicionales de hacer las cosas. A ello se suma la "mirada hacia afuera" que prevalece en la ciencia latinoamericana, supeditada al financiamiento externo, y las demandas de la academia concentrada en publicaciones y producción para el Norte global.

Tampoco se utiliza el idioma originario de las poblaciones, y no se toma en cuenta a las personas más excluidas dentro de las comunidades, por ejemplo, mujeres con cargas domésticas, de tareas de cuidado o laborales (remuneradas o no) que no pueden asistir a asambleas, talleres y otras actividades convocadas por los proyectos. Por último, una vez recolectada la información, no se valida con las comunidades lo que se incluirá en los informes o publicaciones, ni tampoco se devuelve información. En ese sentido, se trata entonces de una ciencia "extractivista" que no beneficia a las comunidades que proveen los datos y que continúan en una situación de exclusión de la producción de conocimiento hegemónico.

\section{GESTIÓN PÚBLICA Y DESARROLLO HUMANO}

El Programa de Naciones Unidas para el Desarrollo (PNUD) define el concepto de desarrollo humano como "[...] la expansión de las libertades de las personas para llevar una vida prolongada, saludable y creativa; conseguir las metas que consideran valiosas y participar activamente en darle forma al desarrollo de manera equitativa y sostenible en un planeta compartido" (PNUD, 
2014, p. 15). Por tanto, el desarrollo humano coloca a las personas en el centro, es inclusivo, equitativo, sostenible y participativo. Asimismo, considera a los individuos como agentes de su propio bienestar, de manera que puedan ampliar sus opciones, alcanzar su potencial y tener la libertad de elegir la vida que valoran (Veltmeyer, 2010, p. 28).

En Latinoamérica estamos aún lejos de alcanzar los estándares de desarrollo humano, previstos primero en los Objetivos del Milenio (2000-2015) y luego en los Objetivos de Desarrollo Sostenible (2015-2030). De acuerdo con el reciente Informe Regional de Desarrollo Humano (PNUD, 2021), los países latinoamericanos son los que presentan mayor desigualdad en el mundo, referida no solamente a ingresos, sino en múltiples indicadores tales como: brechas de género entre participación laboral y tareas de cuidado, discriminación y violencia sobre la población LGBTIQ+, y exclusión de población indígena y otras minorías étnicas. Dicho panorama de desigualdad y exclusión se ha exacerbado con la pandemia por COVID-19 y ha impactado en múltiples ámbitos: violencia doméstica, rezago educativo, pérdida de ingresos y empleos, baja productividad y crecimiento económico, entre otros.

En ese contexto, la gestión institucional e implementación de políticas públicas en Latinoamérica está supeditada a perspectivas con resabios coloniales y estructuralmente racistas y discriminatorios (Solís, 2017). Dicha gestión es también de corte neoliberal, gracias a las medidas impulsadas desde la década de 1980 para reducir el Estado, cuya consecuencia ha sido la disminución de inversión pública en ámbitos sociales (Katz, 2015). Es un sistema que ha excluido histórica y estructuralmente a las poblaciones tradicionalmente consideradas como subalternas o marginadas del desarrollo nacional, tales como afrodescendientes, indígenas, campesinos, jóvenes, mujeres, adultos mayores, personas con discapacidad, población LGBTIQ+, entre otros.

De acuerdo con Solís (2017, p. 25), la discriminación se entiende como "un trato injusto de unas personas hacia otras, en virtud de la pertenencia de estas últimas a un grupo social sobre el cual existen prejuicios u opiniones sociales negativas". Dicho trato injusto se traduce en prácticas, ya sea informales 0 institucionalizadas, que resultan en la privación sistemática o menoscabo en el acceso a derechos y en la reproducción de la desigualdad. Cabe indicar que la discriminación se caracteriza como estructural cuando se sustenta en un orden social independiente de los individuos, a través de la acumulación de desventajas a lo largo de la vida o entre generaciones, con consecuencias macro sociales para el disfrute de derechos y de bienestar (Solís, 2017).

Lo anterior se evidencia en cuánto invierten los Estados latinoamericanos en territorios rurales, en mujeres 0 en indigenas y afrodescendientes, 0 en la disponibilidad de servicios básicos en idiomas originarios y sistemas universales de protección social. Asimismo, en la implementación de programas o políticas homogeneizantes y estandarizadas con resultados muy dispares, y la imposición de iniciativas sin consulta o sin reconocer las dinámicas culturales y reivindicaciones de las poblaciones.

El combate a la discriminación y racismo estructural pasa por el diseño e implementación de políticas públicas y normativas de prevención y garantía de derechos. Asimismo, son necesarios planes, programas y otros mecanismos institucionales que contribuyan con la educación intercultural, mayor sensibilización y espacios para la reflexión y discusión sobre el tema en el ámbito público. Lo anterior requiere de una manera distinta de aproximarnos al Otro, y ello pasa por la perspectiva decolonial.

\section{EL DOSSIER}

Promover la perspectiva decolonial es un desafío. Los dos volúmenes del dossier "Descolonización de la ciencia de los desastres: enfoques desde Latinoamérica y el Caribe" son parte de las iniciativas relacionadas al manifiesto "Poder, prestigio y valores olvidados: un manifiesto de estudios sobre desastres" 1 , iniciado por académicos y activistas de estudios de desastres de todo el mundo. El manifiesto apunta a crear un futuro alternativo donde las epistemologías locales y los conocimientos de los pueblos oprimidos y olvidados puedan tener un espacio adecuado en los estudios de riesgos y desastres.

La convocatoria del dossier de la Revista de Estudios Latinoamericanos sobre Reducción del Riesgo de Desastres (REDER) se inició en agosto de 2020, invitó a autores y autoras que trabajan desde y sobre la región de Latinoamérica y el Caribe, a presentar artículos que promuevan la pluralidad del conocimiento mediante la valoración de las ontologías y epistemologías locales,

1. https://www.ipetitions.com/ petition/power-prestige-forgottenvalues-a-disaster 
cuando corresponda, para descolonizar los estudios de desastres y trascender a los enfoques, fuentes, conceptos, metodologías, valores y lenguajes occidentales 'bien establecidos' que son predominantemente ajenos a los puntos críticos de desastres y riesgos que afectan a la región. Se preparó un listado de temas de interés que incluyeron: pensamiento social latinoamericano ante los desastres y epidemias; descolonización de la ciencia de los desastres; cosmovisiones, conocimiento de los pueblos oprimidos y saberes alternativos en la investigación de desastres; ecología de los saberes; antropología de los desastres; sociología de los desastres; nuevas geopolíticas y biopolíticas en los desastres y pandemias; modelos de desarrollo y producción social de los riesgos de desastres; procesos de vulneración, formas de opresión, desigualdad y pobreza en los desastres; racismo estructural y ambiental en los desastres; desastres olvidados y pueblos oprimidos; capitalismo del desastre; dimensiones del poder en la producción social de los riegos y en los desastres; éticas de investigación de desastres; perspectiva feminista de los estudios de desastres; movimientos sociales y estrategias de resistencia en los escenarios de riesgos y desastres; nuevos abordajes y métodos de investigación; y desafíos de investigación en los enfoques inter y transdisciplinarios. Se recibieron 26 resúmenes en la fase inicial y 14 fueron seleccionados para su publicación en dos volúmenes.

El primer volumen fue compuesto por ocho artículos de Brasil, Chile, Colombia, Ecuador, México y Puerto Rico. El 5 de agosto de 2021 se realizó un lanzamiento virtual coordinado por la Maestría en Desarrollo de la Universidad del Valle de Guatemala y REDER ${ }^{2}$, y el 4 de octubre de 2021 se llevó a cabo un taller en el marco del II Seminario Internacional "Desnaturalização dos Desastres e Mobilização Comunitária: crises ampliadas, redes e resistências" ${ }^{3}$, con participación de 72 personas $^{4}$ (Figura 1).

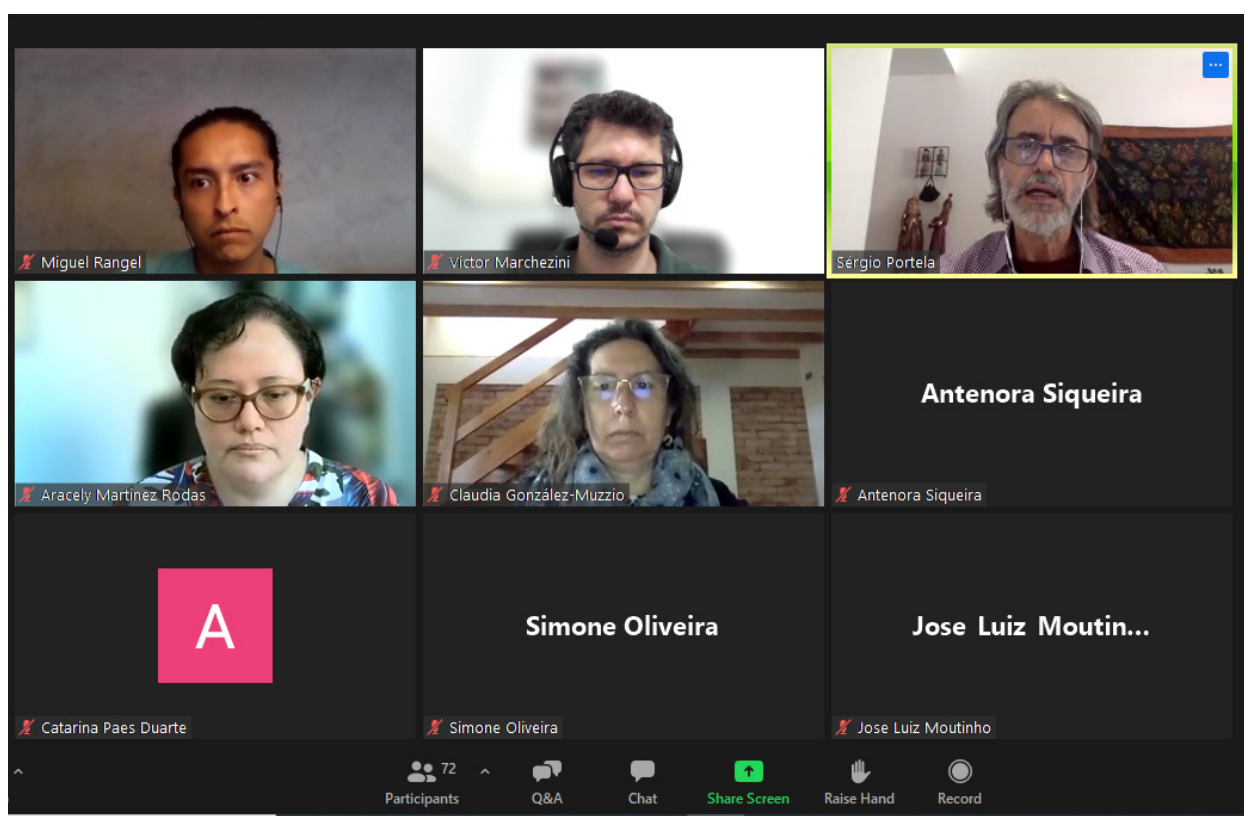

Figura 1. Taller sobre "Descolonización de la Ciencia de los Desastres", durante el II Seminario Internacional sobre Desnaturalización de la Ciencia de los Desastres (4 octubre de 2021). Fuente: Autores, 2022

Los artículos del volumen 1 del dossier abarcaron los discursos y pensamientos de las culturas mesoamericanas, reflexiones teóricas y metodológicas desde una perspectiva críticafeminista, pensamiento decolonial latinoamericano, críticas al enfoque técnico-científico y militar en la gestión de riesgos, así como a la trayectoria colonial interna de la gestión de riesgos, subordinada a los intereses políticos y económicos de los gobiernos y élites de turno, prolongando formas del colonialismo interno, en clave étnica y de género, a las poblaciones locales, indígenas campesinas y afroecuatorianos. El enfoque histórico también es una dimensión clave en los artículos, así como el análisis de los procesos contemporáneos del modo neoliberal del gobierno de desastre y los juegos de poder en el territorio, con el control de las memorias y narrativas y proposición de soluciones estandarizadas. Todavía existen las estrategias comunitarias y metodologías participativas de investigación-acción, como una contribución al agenciamiento y al mayor énfasis del conocimiento situado transdisciplinar, siendo un potencial vehículo movilizador para expandirse las formas de
2. El video del evento puede verse en el siguiente enlace: https://www. facebook.com/100057399942723/ videos/4065560843572553/

3. Enlace del II Seminario: https:// youtu.be/2ijaacuohyI

4. Nuestro agradecimiento a Sergio Portella, Simone Oliveira, Antenora Siqueira y todos(as) de la Comisión Organizadora del evento por la invitación para coordinar el taller. 
resistencia a partir de voces subalternas y promover la noción de dignidad como categoría moral para la transformación, dejando de lado ideas de estabilidad y despolitización.

El primer volumen invita a reflexionar sobre la importancia de tres elementos interrelacionados (Figura 2): por un lado, el conocimiento situado sobre los eventos climáticos y geológicos que tienen las poblaciones. Esto se refiere a cómo diferentes grupos y culturas crean y perciben las dinámicas naturales que ocurren en sus territorios y se expresan en prácticas, mitos, explicaciones, percepciones, actitudes y comportamientos socioculturales.

En segundo lugar, la gestión institucional para la prevención y manejo de desastres está supeditada a una perspectiva colonial, neoliberal y estructuralmente racista, que continúa excluyendo a grupos tradicionalmente marginados: afrodescendientes, indígenas, campesinos, jóvenes, personas mayores y mujeres. De esta manera, las respuestas institucionales, ya sea para la atención, manejo o prevención de los desastres, no son pertinentes culturalmente y perpetúan las vulnerabilidades de dichas poblaciones.

En tercer lugar, se pone énfasis en la urgencia de una perspectiva decolonial y feminista que incluya poblaciones excluidas, que permita negociaciones más equitativas en las relaciones de poder entre poblaciones e instituciones, que dé voz a las mujeres y otras minorías. En ese sentido, la gestión y reducción de riesgos y desastres requieren de políticas públicas más integrales, con un enfoque interseccional, de prevención y protección social, donde los protagonistas sean las familias y comunidades desde sus prioridades de atención diferenciadas.

\section{Políticas públicas}

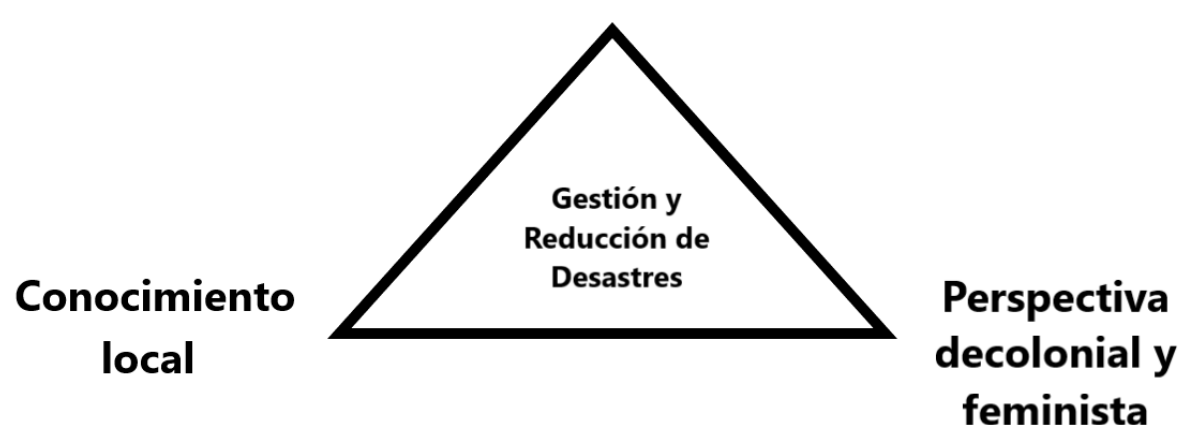

Figura 2. Tres elementos interrelacionados del proceso de descolonización de la ciencia de los desastres Fuente: Marchezini et al. 2021

El volumen 2 del dossier promueve la continuación del debate del primer volúmen. Está integrado por seis artículos de México, Brasil, Venezuela, Costa Rica y Colombia. Inicia con el artículo "Neo Colonialidad'y gestión del riesgo de desastres en América Latina", de Jesús Manuel Macías. Para el autor, el examen del desarrollo histórico de las organizaciones que los gobiernos en el continente americano han desplegado para enfrentar desastres muestra diferencias notables entre Estados Unidos (y Canadá) y el resto de los países latinoamericanos. Esas diferencias pueden ser observadas tanto en la configuración de las administraciones públicas, en término de modelo de organización (Defensa Civil/Protección Civil, Manejo de Emergencias), como en las bases conceptuales del proceso de desastre con fines de intervención (fases del desastre) y la reinvención artificial de los conceptos riesgo, y resiliencia. Esas diferencias suponen una relación de colonialidad ampliamente reforzada, donde se mantiene evidente el rol hegemónico directo (en la relación bilateral y multilateral), e indirecto (a través de las agencias de la ONU incluidas las financieras como el Banco Mundial).

Este proceso se refleja en la producción de formas subordinadas de conocimiento, lo que requiere revisiones sistemáticas sobre cómo se generan los saberes en el contexto latinoamericano. En el artículo "Enfoques hegemónicos en la gestión del riesgo de desastres asociados a eventos hidrometeorológicos: análisis del contexto de Latinoamérica y el Caribe", de Miguel Angel TrejoRangel, Rodrigo Rudge Ramos Ribeiro, Cristian Camilo Fernández Lopera, Adriano Mota Ferreira, Nhilce Nahomi Esquivel-Gomez y Carla Cristina Liera-Martínez, se busca identificar cuáles son los 
enfoques de intervención (específicamente de arriba hacia abajo) que predominan en la literatura científica en portugués y español, entre los años 2015 a 2020. Los resultados muestran que la mayoría de los artículos (82\%) se basan en el enfoque de arriba hacia abajo, sólo el $13 \%$ hace uso de métodos participativos, y con un énfasis en procesos de prevención (57\%), preparación (41\%), respuesta (36\%) y/o recuperación $(21 \%)$.

Para contribuir con la decolonización del enfoque analítico eurocéntrico de las catástrofes, en el artículo "Vulnerabilidad socioambiental desde los feminismos comunitarios. El desastre por el sismo del 19s en Tetela del Volcán, Morelos, México", Alejandra González-Ramírez, Frida Güiza, Martha Shirley Reyes-Quintero, Maria Elena Méndez-López, y Pablo Torres-Lima, plantean construir puentes de diálogo entre el marco de análisis de la vulnerabilidad socioambiental proveniente de la ecología política y los feminismos comunitarios de las Epistemologías del Sur, con el fin de proponer perspectivas latinoamericanas descolonizadas. De acuerdo con los resultados del caso de estudio, los feminismos comunitarios nombran las violencias estructurales que emergen durante la catástrofe, construidas históricamente sobre el cuerpo de las mujeres y la naturaleza. Además, visibilizan el trabajo comunal de las mujeres para la atención y el cuidado en la práctica cotidiana. El estudio destaca la Comunidad como categoría política de acción, resistencia y emancipación en el cuerpo-territorio-tierra que conlleve a la disminución de su vulnerabilidad socioambiental ante el riesgo de desastre.

¿Pero, cuáles son las dimensiones centrales para la disminución de la vulnerabilidad socioambiental? Los diferentes contextos y grupos sociales pueden indicar respuestas variadas. En el artículo "Pobreza, desigualdad social y vulnerabilidad a los desastres en Venezuela", de Bettys Farias, Adriana Marquez, y Edilberto Guevara, el objetivo de la investigación es proponer la noción de la pobreza, la desigualdad social y la privación, como elementos que causan vulnerabilidad a los desastres, demostrando la forma en la que la construcción social del riesgo se relaciona directamente con las políticas públicas nacionales de acceso a viviendas, empleos, y ordenamiento territorial. Los autores reportan que de acuerdo a la Encuesta Sobre Condiciones de Vida en Venezuela (Encovi 2019-2020) se revela que la caída de 70\% del Producto Interno Bruto (PIB), acumulada en los últimos siete años, deja a su paso $96,2 \%$ de la población en pobreza por ingresos, y a 79,3\% en pobreza extrema que no pueden cubrir la cesta básica, con una inflación acumulada entre marzo de 2019 y marzo de 2020 que se ubica en $3365 \%$. La crisis sanitaria por el COVID-19 ha generado que hasta $43 \%$ de los hogares reporten imposibilidad de trabajar o pérdida de ingresos. Los hogares que dijeron que trabajaron más horas pasó de $41 \%$ a $49 \%$, quienes pidieron dinero a sus familiares pasó de $30 \%$ a $35 \%$. Dentro de este contexto, la investigación aplicó 715 encuestas entre las 6 comunidades, para evaluar las necesidades básicas (educación básica, vivienda adecuada, disponibilidad de agua y acceso a servicios sanitarios básicos, capacidad económica) satisfechas (NBS) e insatisfechas (NBI), y también producir mapas de riesgo de desastres con apoyo de la comunidad. Otro aspecto destacado en esta investigación es la posición de Venezuela frente al cumplimiento de los Objetivos del Desarrollo Sostenible (ODS): no se reportan avances de su gestión desde 2016, ni se han definidos mecanismos de información de avances, lo cual es preocupante porque demuestra la inactividad para cumplir al menos con disminuir la pobreza extrema que a su vez favorece la disminución de la vulnerabilidad.

Los datos para monitoreo de estos avances y retrocesos en los ODS, así como de las necesidades básicas, de la vulnerabilidad, de las capacidades y de los impactos de los desastres también son importantes, tanto como la política de creación de los datos, sus formas de recolección y circulación. Muchas veces la política de dominación colonial también ocurre a través de los datos y de los umbrales establecidos, con situaciones en que algunos países y empresas tienen más datos e informaciones sobre los países de Latinoamérica y el Caribe que ellos mismos. Los desastres a pequeña y mediana escala suelen excluirse de las bases de datos globales sobre desastres, pero pueden tener consecuencias corrosivas para las vidas y los medios de subsistencia, sobre todo en las comunidades más pobres y vulnerables, como lo ejemplifica el artículo sobre el caso de Venezuela, en este segundo volumen del dossier. Para tener autonomía sobre su proceso de producción de datos, La Red de Estudios Sociales en Prevención de Desastres en América Latina (La Red) empezó a crear, en 1993, la herramienta teórica y metodológica DesInventar, que recopila registros históricos de pérdidas de desastres y facilita el análisis y la representación espacial y temporal de amenazas, vulnerabilidades y riesgos de manera retrospectiva y prospectiva de utilidad para la gestión del riesgo y otros ámbitos de gestión del desarrollo. Debido al grado 
de detalle que aporta este tipo de datos de la herramienta DesInventar, resulta posible registrar las pérdidas derivadas de una serie de fenómenos recurrentes a pequeña y mediana escala que producen y acumulan daños, lo que permite calcular lo que se conoce como "riesgo extensivo". En el artículo "Inventario histórico de desastres en Costa Rica en el periodo 1970-2020", Ricardo A. Orozco-Montoya, Alice Brenes-Maykall y Rebeca Sura-Fonseca caracterizan las tendencias y comportamientos de los pequeños, medianos y grandes desastres en Costa Rica registrados en la base de datos DesInventar. A partir del 2011 y hasta la actualidad, el proyecto ha logrado mantener la base de datos de Costa Rica actualizada a través de una actividad académica del Programa Institucional de Gestión del Riesgo de Desastre (PIGRD) de la Vicerrectoría de Extensión de la Universidad Nacional (UNA) de Costa Rica. Los recortes presupuestarios al sistema de educación superior pública costarricense atentan contra la acción sustantiva universitaria, en este caso, en el mantenimiento de la base de datos DI-Costa Rica, que posee un total de 19.016 registros de eventos ocurridos en el país en el periodo 1970-2020. Del total de eventos registrados en el periodo 1970-2020 (19.016), el 80\% corresponde a eventos de tipo hidrometeorológico, 13\% a epidemias y eventos humano-tecnológicos y $7 \%$ a geológicos, lo que ha registrado 1.555 personas fallecidas. Los distritos clasificados con bajo y muy bajo desarrollo relativo concentran el $40 \%$ de eventos registrados.

La búsqueda de datos históricos acerca de las amenazas, vulnerabilidades, desastres e impactos, mediante información que pueda proporcionar noticias y bases de datos también es un paso fundamental para plantear medidas de reducción de riesgos. En el artículo "Integración de conocimientos para reducir riesgos por derrames de hidrocarburos en fuentes hídricas", Verónica Zapata Gómez, Manuela Cortés Cano, Olga Lucía Amaya Moncada, Laura Osorio Cano y Edna Margarita Rodríguez Gaviria, utilizan una serie de metodologías cuantitativas y cualitativas para el análisis de fuentes de amenaza, del mapa de actores, para cartografiar las afectaciones y formular estrategias para medidas correctivas y prospectivas en la reducción del riesgo.

Las autoras crearon un mapa de las zonas afectadas y una línea del tiempo de la ocurrencia de derrames de hidrocarburos en fuentes hídricas en el Corregimiento de San Cristóbal, Medellín, Colombia. A partir de esto se realizó un mapa conceptual con el fin de identificar las fuentes que generan amenaza a la prestación del servicio de agua potable en la zona por motivo de derrames, explosiones del oleoducto, acciones delictivas, así como de los diferentes actores dentro del corregimiento de San Cristóbal y cómo estuvieron involucrados en la problemática presentada. A través del mapa de actores también fue posible observar los diferentes grupos relacionados con la gestión del riesgo de acuerdo con su presencia territorial correspondiente a las escalas nacional, regional y local y una clasificación por niveles: primer nivel, aquellos que producen o centralizan la información (incluyendo políticas) y la transmiten a otras partes; de segundo nivel, los principales interesados en la gestión de emergencias, y por lo general, obtienen la información y las instrucciones de las instituciones de primer nivel. Un tercer nivel son aquellas instituciones públicas que desempeñan un papel importante en la planificación estratégica, tal como los del primer nivel y actúan como un puente para la comunicación de la gestión del riesgo entre las autoridades locales y el público en general. En el cuarto nivel, miembros de la comunidad, organizaciones comunitarias, instituciones públicas y privadas, los cuales son propensos a ser afectados y que se convierten en los principales beneficiarios de una adecuada gestión del riesgo. Además, se incluyeron las funciones que cumplen cada uno de estos en el corregimiento de San Cristóbal.

El artículo también comparte los resultados de una encuesta con los moradores y entrevistas con organismos locales. Los resultados de la investigación fueron compartidos en una capacitación virtual para identificar cuándo el agua del grifo está contaminada por hidrocarburos, debido a los cambios en sus características (olor, sabor, color), las posibles afectaciones a la salud y cómo actuar en caso de ingerir agua con hidrocarburos. El propósito es una gestión del riesgo oportuna para la comunidad afectada, para la pronta acción ante la ocurrencia del derrame y contacto comunitario con fuentes hídricas contaminadas con hidrocarburos. El artículo recomienda acciones para mejorar la gestión correctiva y prospectiva de los riesgos, incluyendo aquellos tipos de amenazas que son olvidadas en las políticas públicas. 


\section{EDITORES INVITADOS:}

Aracely Martínez Rodas, antropóloga, Guatemala.

Claudia González-Muzzio, arquitecta, Chile.

Victor Marchezini, sociólogo, Brasil.

\section{REFERENCIAS}

Katz, C. (2015) “Peculiaridades del neoliberalismo en América Latina”, Pacarina del Sur, 7(25), 1-18. http://hdl.handle.net/11336/72444

Marchezini, V., González-Muzzio, C. \& Martínez Rodas, A. (2021). Descolonización de la ciencia de los desastres: enfoques desde Latinoamérica y Caribe. Revista de Estudios Latinoamericanos sobre Reducción del Riesgo de Desastres REDER, 5(2), 1-4. http://revistareder.com/

handle-0719-8477-2021-120

Programa de Naciones Unidas para el Desarrollo (PNUD). (2014). Sinópsis del Desarrollo Humano en Guatemala. Guatemala: PNUD. http://desarrollohumano.org.gt/sites/default/files/sinopsis_ desarrollo_humano.pdf

Programa de Naciones Unidas para el Desarrollo (PNUD). (2021). Atrapados: Alta desigualdad y bajo crecimiento en América Latina y el Caribe. Informe Regional de Desarrollo Humano. Nueva York: PNUD. https://www.latinamerica.undp.org/content/rblac/es/home/library/human_development/ regional-human-development-report-2021.html

Santa, S. \& Herrero Solana, V. (2010). Producción científica de América Latina y el Caribe: Una aproximación a través de los datos de Scopus, 1996-2007. Revista Interamericana de Bibliotecología, 33(2), 379-40o. http://ref.scielo.org/xtmjh3

Solís, P. (2017). Discriminación estructural y desigualdad social: Con casos ilustrativos para jóvenes indígenas, mujeres y personas con discapacidad. México: Consejo Nacional para Prevenir la Discriminación.

Veltmeyer, H. (2010). Una sinopsis de la idea de Desarrollo. Migración y Desarrollo, 8(14), 9-34. http:// ref.scielo.org/sybx6m 